\title{
On the assembly of Archimedean spiral cavities for sound absorption applications: design, optimization and experimental validation
}

\author{
Carvalho De Sousa, Augusto ${ }^{1,2, *}$, Deckers, E. ${ }^{2,3}$, Claeys, C. ${ }^{1,2}$, Desmet, W. ${ }^{1,2}$ \\ ${ }^{1}$ Department of Mechanical Engineering, KU Leuven, Belgium, \\ Celestijnenlaan 300, 3001 Leuven, Belgium \\ ${ }^{2}$ DMMS lab, Flanders Make, Belgium \\ ${ }^{3} \mathrm{KU}$ Leuven, Diepenbeek Campus, Mechanical Engineering Technology TC, \\ Wetenschapspark 27, 3590 Diepenbeek, Belgium \\ *Corresponding author: augustocarvalho@tutanota.com
}

\begin{abstract}
This work presents the design, optimization and combination of fully parameterized Archimedean spiral cavities (ASCs) as acoustic resonators, in order to achieve perfect absorption at frequencies below $1000 \mathrm{~Hz}$ while using a completely modular design. The assembly of two ASCs in the same system is also presented. An optimization is performed and results are verified through Finite Element Analysis (FEA) and validated experimentally for almost perfect absorption peaks at $700 \mathrm{~Hz}$ and $800 \mathrm{~Hz}$. A good agreement is achieved between theory, simulation and experiments.
\end{abstract}

Keywords: Archimedean spiral cavities, Metasurfaces, Acoustic absorption

\section{Introduction}

Acoustic treatment of rooms is required to diminish noise coming from machinery and other sources [1]. Acoustic treatments are also applied to make the listener's experience more pleasant [2] in case the room is devoted to entertainment, such as listening to music. Sound absorbing materials and structures are often applied to obtain an optimal reverberation time in a pre-determined range of frequencies. Low frequency absorption proves to be difficult as the thickness of the absorbing material needs to be larger than $\lambda / 4, \lambda$ being the wavelength of the sound wave at a particular frequency, in 
order to provide high absorption coefficients [3]. If foams or fibrous materials are to be applied, this leads to bulky and impractical solutions.

Micro-perforated panels (MPPs) backed by an air cavity are widely applied to obtain high sound absorption coefficients at low frequencies $[4,5$, $6,7]$. The small holes provide high viscous losses, but, as for foams and wools, the depth of the backing cavity becomes large to be effective at lower frequencies.

Helmholtz resonators (HRs) have also been used in literature [8, 9] as acoustic absorbers, but only provide a good absorption coefficient in a small frequency band, thus limiting their application for broadband absorption.

Coiled channels and spirals are structures that can provide a high absorption coefficient at low frequencies while keeping the thickness of the structure in the deep-subwavelength scale. These show great potential for practical applications in room acoustics and noise control [10]. This approach for sound absorption first appeared in literature in the work of Cai et al. [10], whose structure has a thickness of $\lambda / 102$. The frequency at which the absorption peaks occur is mainly determined by the length of the channel. Spiralled channels are also used in the work of Li et al. [11], who combine an MPP and a coiled space in order to achieve a thickness of $\lambda / 223$. Their theory is verified by numerical simulations; no experiments are performed. Further improvements are made by Huang et al. [12], who combined an embedded aperture with a coiled channel to achieve more tunability in their system, which resulted in a thickness of $\lambda / 100$ at very low frequencies. All of these strategies provide a good ratio of thickness to $\lambda$, but the absorption peak is very narrow and perfect absorption only occurs at a single frequency due to the natural resonance of the coiled channel. Chen et al.[13] designed a spiralled channel with different cross section areas along its segment, achieving two peaks of absorption, at $700 \mathrm{~Hz}$ and $800 \mathrm{~Hz}$, respectively, within a thickness of $\lambda / 38.5$. Between those frequencies, the absorption coefficient remains higher than 0.8. Multiple coiled spaces based on the theory of HRs also have been studied $[14,15]$, with the system being optimized accounting for more than one HR at once. Combination of MPPs and HRs [16, 17], arrays of HRs with different dimensions $[18,19]$ and honeycomb cells that mimic the behaviour of HRs [20] have also been tested to target a broader frequency range.

Structures with more than one coiled channel, such as Fabry-Perot acoustic cavities, are present in literature [21]. The channels are optimized following the causality constraint, which links frequency-dependent absorption coeffi- 
cient to sample thickness. The authors demonstrate the theory with a design with a thickness of only $10.56 \mathrm{~cm}$, which consists of 16 channels, and gives a high sound absorption coefficient starting from $400 \mathrm{~Hz}$ onwards. The thickness of the sample is thus $\approx \lambda / 10$. Combinations of HRs specifically tuned for a broadband absorption also have been applied in the so-called rainbow trapping absorbers [22]. The HRs are loaded in an array of varying cross section waveguides, and the low cut-off frequency is determined by the resonance frequency of the largest HR. By adding different HRs to the waveguide, a cascade effect is obtained and quasi-perfect absorption is reported from 300 $\mathrm{Hz}$ to $1000 \mathrm{~Hz}$. The structure has a thickness of $\approx 11 \mathrm{~cm}$, thus being 10 times smaller than the wavelength at $300 \mathrm{~Hz}$.

Inspired by the aforementioned works, in this paper a strategy is presented to combine different resonators, namely Archimedean spiral cavities (ASCs), that are embedded in the same geometry in order to provide sound absorption peaks in more than one frequency. Each resonator is optimized for a targeted frequency and manufactured in a way that makes it fully independent from the others, while making the system modular at the same time. Special attention is paid to their design, allowing for a straightforward production by 3D-printing process. The strategy is verified numerically through Finite Element Analysis (FEA) and validated with experiments in an impedance tube.

This work is divided as follows: Section 2 presents the theoretical models used for the geometry; Section 3 presents the design and numerical simulation processes; Section 4 discusses the results and Section 5 draws the main conclusions.

\section{Models and methods}

This section presents (i) the parameterization of the Archimedean spiral cavities, (ii) the analytical model to account for sound absorption in these channels and (iii) the combination of more than one channel in the same geometry.

\subsection{Parameterization of the Archimedean spiral cavities}

Archimedean spiral cavities provide a thin geometry for sound absorption in low frequencies due to space coiling. In addition, the full parameterization ensures that the geometry can be straightforwardly optimized to obtain a 
high absorption coefficient at targeted frequencies. A schematic representation of such a spiral is found in Figure 1.

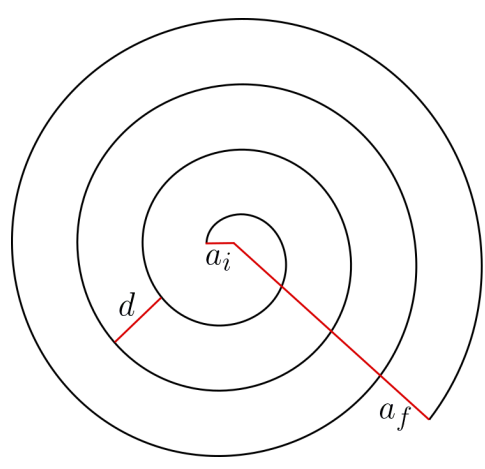

(a)

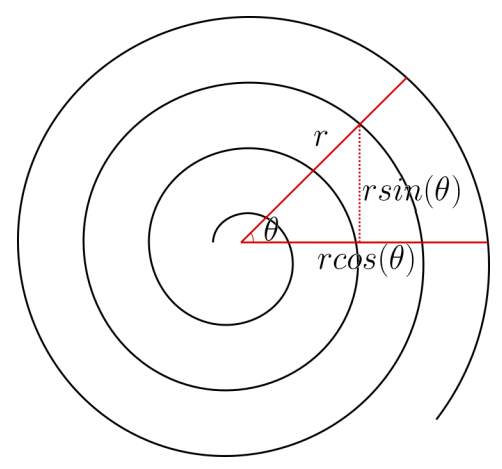

(b)

Figure 1: Archimedean spiral (a) and parameters in polar coordinates (b).

Equation (1) gives the spiral radius $r$ as a function of the initial radius $a_{i}$ and the angle $\theta$ :

$$
r=a_{i}+b \theta
$$

For the final angle $\theta=\theta_{f}$, the final radius is $r=a_{f}$. The $x$ and $y$ components of $r$, as a function of polar coordinates, are

$$
x=r \cos (\theta)
$$

and

$$
y=r \sin (\theta) .
$$

The parameter $b$ is the spiral growth ratio, which is given by

$$
b=\frac{a_{f}-a_{i}}{2 \pi k},
$$

where $a_{f}$ is the final radius and $k$ is the number of turns of the spiral.

The distance $d$ between successive turns is then

$$
d=2 \pi b .
$$

The spiral shown in Figure 1 is a 1-dimensional (1D) curve, which can be expanded into a 2D geometry as seen in Figure 2, thus becoming a spiralled 
channel whose $2 \mathrm{D}$ cross section has a thickness $t$. The distance between turns $d$ is dependent on the thickness $t$ and on the thickness of the wall $d_{\text {wall }}$ : $d=d_{\text {wall }}+t$. Finally, it is possible to turn the $2 \mathrm{D}$ channel into a $3 \mathrm{D}$ cavity by assigning a height $h$, in case the cross section is rectangular, or a radius $r_{t}=t / 2$ in case the cross section is circular.

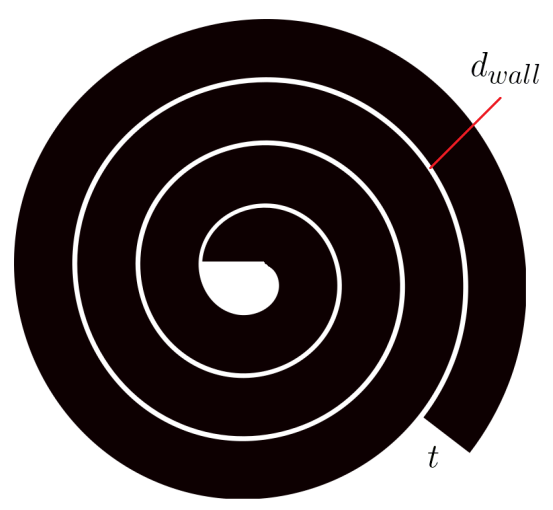

Figure 2: Spiral with expanded sides with thickness $t$ and distance between turns $d_{\text {wall }}$.

Finally, the final angle of the spiral can be written as

$$
\theta_{f}=\frac{a_{f}-a_{i}}{b}
$$

The total length of the spiral is then determined by [23]:

$$
L_{A S C}=\int_{\theta_{i}=0}^{\theta_{f}} \sqrt{a_{f}^{2}+\left(d a_{f} / d \theta\right)^{2}} d \theta .
$$

\subsection{Acoustic impedance calculation}

An ASC (Figure 3) whose front panel has area $A$, has an impedance of

$$
Z_{A S C}=-j Z_{c}\left(A / s_{0}\right) \cot \left(k_{e f f} L_{A S C}\right),
$$

where $s_{0}$ is the cross section area of the ASC and $Z_{c}=\left(\rho_{e f f} K_{e f f}\right)^{1 / 2}$ is its characteristic impedance [24]. $\rho_{\text {eff }}$ and $K_{\text {eff }}$ are the effective density and effective compressibility, respectively [24, 2], and the effective wavenumber is given by $k_{\text {eff }}=\omega \sqrt{\rho_{\text {eff }} / K_{\text {eff }}}$. 

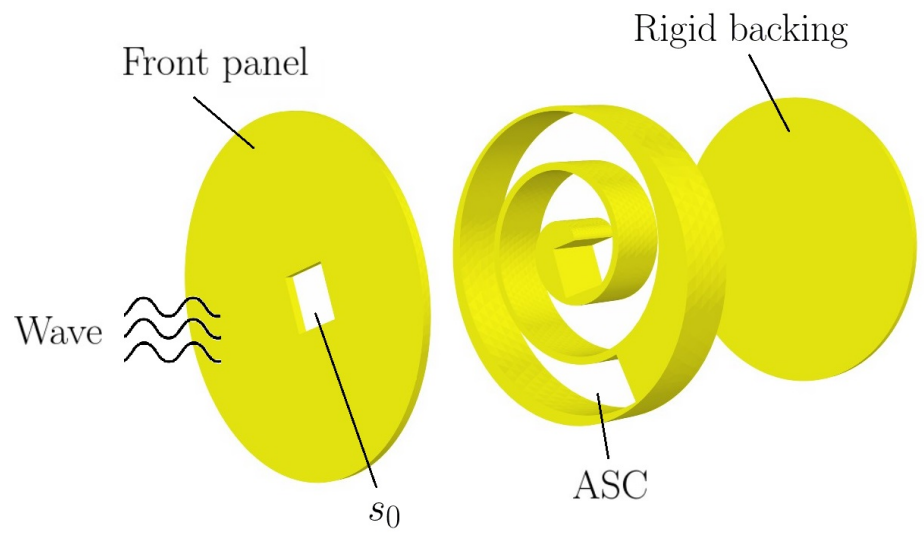

Figure 3: Unit cell with one ASC and a rectangular cross section.

The characteristic impedance $Z_{c}$ of a channel, whether it is a spiral or any other geometry, is dependent on its cross sectional shape, since the effective density $\rho_{\text {eff }}$ and compressibility $K_{\text {eff }}$ are cross section dependent. Circular cross sections are shown to perform better, i.e. thinner sample thickness and high absorption, than rectangular ones [24]. For a channel with a circular cross section of radius $r_{t}$, the effective density and compressibility are given by $[25]$ :

$$
\rho(\omega)_{e f f}=\rho_{0}\left[1-\frac{2}{r \sqrt{-i \omega \nu}} G(\xi)\right]^{-1}
$$

and

$$
K(\omega)_{e f f}=\left(\gamma P_{0}\right)^{-1}\left[1+\frac{2(\gamma-1)}{r \sqrt{-i \omega \gamma \nu^{\prime}}} G\left(\xi^{\prime}\right)\right],
$$

where $G(\varepsilon)=J_{1}(\varepsilon) / J_{0}(\varepsilon), J_{i}$ is the Bessel function of the $i$-th order, $\rho_{0}$ and $P_{0}$ are the air density and pressure, respectively, $\gamma=1.4$ is the specific heat ratio and $\nu$ and $\nu^{\prime}$ are the dynamic and thermal viscosity of air, respectively; $\xi=r \sqrt{-i \omega / \nu}$ and $\xi^{\prime}=r \sqrt{-i \omega \gamma / \nu^{\prime}}$.

\subsection{Sound absorption coefficient}

In a system with more than one ASC, the resonators must be combined in such a way that they do not interact with each other, i.e. each spiral is isolated from the other by solid walls. An example of assembly is given in Figure 4, considering the combination of 2 ASCs. 


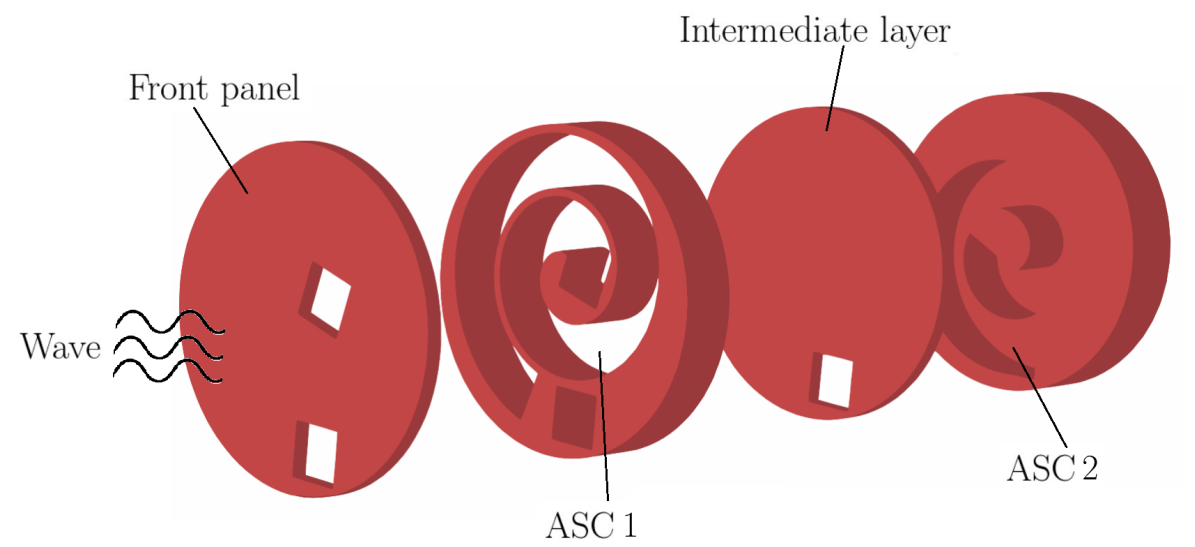

Figure 4: System containing 2 ASCs. The wave enters both ASC 1 and ASC 2 channels at the same time through a front panel with a circular cross section of total area $A$.

Since the systems are being designed targeting frequencies whose wavelength is much higher than the distance between the entrance of the channels, and accounting for the fact that each resonator works in a deep subwavelength frequency, it can be considered that the absorptive effect of one resonator will not be affected by the presence of the other [21]. For parallel assemblies of $N$ different systems, each one with impedance $Z_{N}$, the total impedance is then given by $[24]$

$$
\frac{1}{Z}=\sum_{k=1}^{N} \frac{1}{Z_{k}} .
$$

From Equation (11), it is then possible to calculate the total sound absorption coefficient $\alpha$ as

$$
\alpha_{A S C}=1-\left|\frac{Z-\rho_{0} c_{0}}{Z+\rho_{0} c_{0}}\right|^{2} .
$$

\section{Design and numerical analysis}

This section presents the optimization procedure to obtain dimensions that lead to a maximum absorption peak at a chosen frequency. The numerical modelling of the system is presented, as well as the the manufacturing process. For the latter, 3D-printing is chosen, although other techniques, such as laser cutting, could also be used. 


\subsection{Optimization}

Being simply a coiled channel, the ASC will have a main resonance frequency and its harmonics [2]. In order to find the optimum parameters to obtain unity absorption at a chosen frequency, an optimization procedure is performed with the solver fmincon() from MATLAB. The constraint equation $c(x)$ in the solver is set as

$$
c(x)_{\text {circ }}=a_{f}+r_{t}-(D / 2-0.1) \leq 0,
$$

thus stating that the radius $D / 2$ of the sample must be bigger than the outer spiral radius $a_{f}$ plus the cross section radius $r_{t}$ of the chanel, in case of a circular cross section, leaving $0.1 \mathrm{~mm}$ of gap between the outer radius of the spiral and the edge of the sample. This gap is a tolerance implemented into the solver foreseeing experimental testing, i.e. if the tube has a diameter of $40 \mathrm{~mm}$, it is necessary for the physical sample to have a maximum diameter of $39.8 \mathrm{~mm}$, as a warranty that it will fit into the tube for the experiment to be done. The function to be minimized, since the target is a single frequency and not a range of frequencies, is $f(x)=-\alpha_{A S C}$. Other functions, such as $f(x)=1 / \alpha_{A S C}$, could be used, but, in the case of a system with many resonators, the division operation makes the optimization algorithm more computationally expensive.

\subsection{Numerical simulation}

The numerical simulation of the optimized UC is performed in COMSOL Multiphysics ${ }^{\circledR}$ by means of a Finite Element Analysis (FEA), using the Pressure Acoustics Module with a Frequency Domain study. The spiralled shapes are modelled using Solid Edge software and imported as an .igs file. For each ASC, a Narrow Acoustic Region is assigned as the domain and the type of tube is set as Circular Tube. To excite the channels, a cylindrical air cavity with a diameter of $40 \mathrm{~mm}$ is created and excited on the boundary opposite to the ASC, with a unit pressure to generate a plane wave. For the tube domain, the fluid model is set as Thermally Conducting and Viscous. The whole system is meshed with Free Tetrahedral elements. The element length is verified to be smaller than one sixth of the smallest wavelength. The mesh

consists of 68420 degrees of freedom. A representation of the meshed system is shown in Figure 5. 


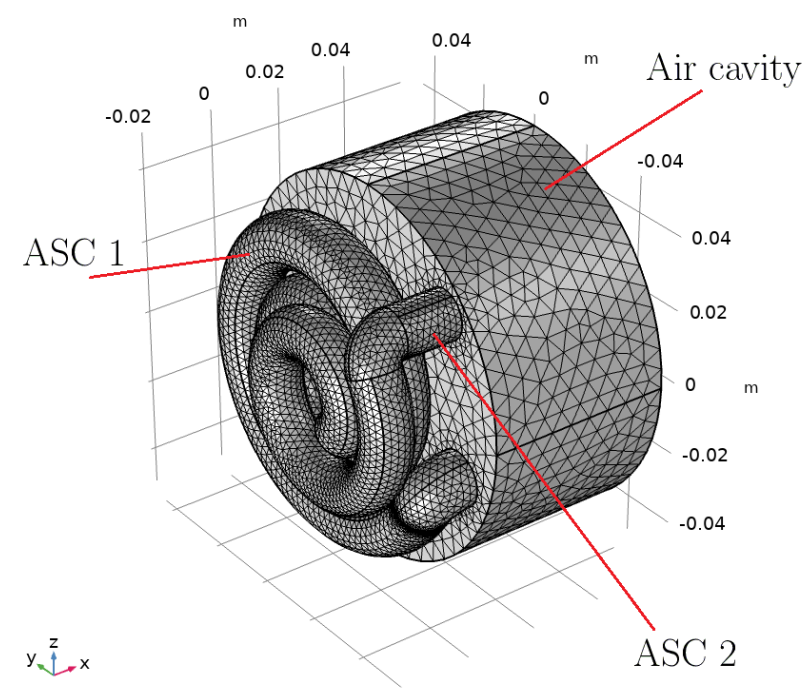

Figure 5: Implementation of the 2ASC system in COMSOL software for a FEA analysis.

\subsection{D-Printing and experiments}

To provide a modular design, the geometry is 3D-printed in different parts, as shown in Figure 6. This allows not only for the removal of the supporting material left by the printing process, but also for an easy assembly of the parts. The channels are printed with a square cross section with sides of size $2 r_{t}$, thus keeping the hydraulic radius the same. As long as the hydraulic radius is maintained, the change from circular to square cross sections can be made without compromising the results for sound absorption [24].

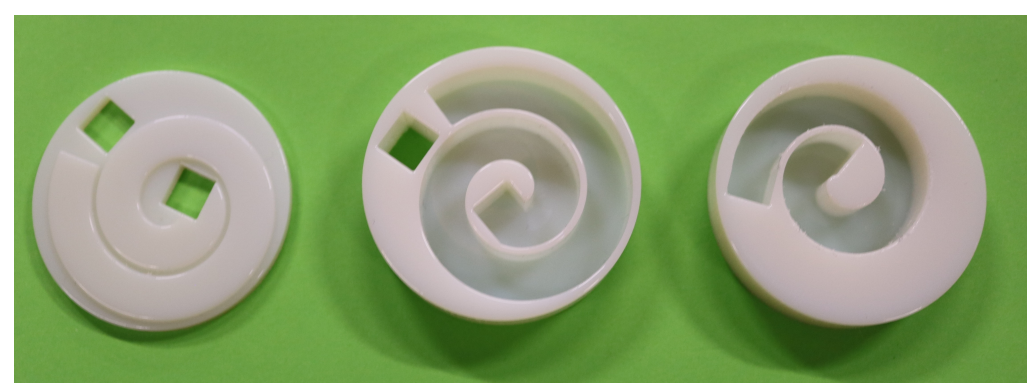

Figure 6: 3D-printed layers of the 2ASC system.

The sample is manufactured using a Objet30 Prime printer, using approximately $35 \mathrm{ml}$ of the material Vero White RGD835. The layers are as- 
sembled together with superglue. The experimental test follows ISO 10534, in an impedance tube with diameter $D=40 \mathrm{~mm}$. The test setup and its schematic representation are represented in Figure 7.

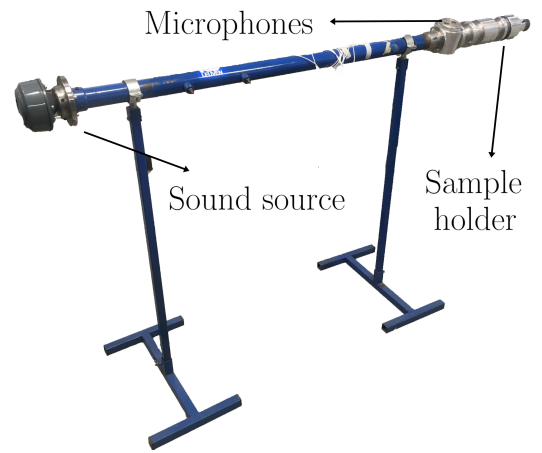

(a)

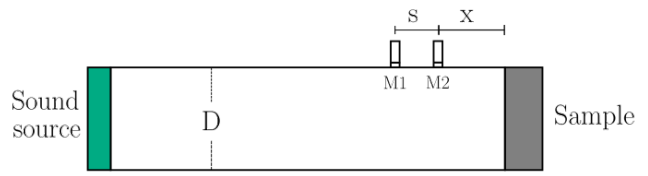

(b)

Figure 7: Impedance tube used for experiments: (a) setup used and (b) schematic representation.

The acoustic cavity is excited via a sound source at the left end of the tube. The pressure variation in the medium is captured by microphones $M 1$ and $M 2$, spaced $s=35 \mathrm{~mm}$ from each other. Microphone $M 2$ is $x=290 \mathrm{~mm}$ away from the sample, which is rigidly backed. The test rig is connected to a SCADAS mobile acquisition system. The data is processed with LMS TestLab software, using the "Measuring Sound Absorption with an Impedance Tube module". The frequency response is calculated up to $6400 \mathrm{~Hz}$ with a frequency resolution of $1.25 \mathrm{~Hz}$ and using 50 averages per run.

\section{Results and discussion}

This section presents and discusses the results of the optimization, numerical implementation and experimental validation.

\subsection{Design and optimization results}

A system containing $2 \mathrm{ASCs}$, namely $\mathrm{ASC}_{1}$ and $\mathrm{ASC}_{2}$, is optimized for absorption peaks at $700 \mathrm{~Hz}$ and $800 \mathrm{~Hz}$, respectively. Each channel is optimized separately as it is considered that there is no interaction effect between them. The frequencies are chosen considering the diameter of the tube available for testing, which is $40 \mathrm{~mm}$. The lower boundary (LB), upper boundary 
(UB) and initial guesses (IG), as well as the optimization results, are shown in Table 1.

Table 1: Optimization parameters and boundaries for the 2ASC system.

\begin{tabular}{c|ccccc|c}
\hline \multirow{2}{*}{$A S C$} & Parameter & LB & UB & IG & Result & $L_{A S C}$ \\
\hline \multirow{2}{*}{$A S C_{1}$} & $a_{f}$ & $10 \mathrm{~mm}$ & $49 \mathrm{~mm}$ & $10 \mathrm{~mm}$ & $17.5 \mathrm{~mm}$ & \multirow{2}{*}{$120.1 \mathrm{~mm}$} \\
& $r_{t}$ & $3 \mathrm{~mm}$ & $25 \mathrm{~mm}$ & $6 \mathrm{~mm}$ & $3.4 \mathrm{~mm}$ & \\
\hline \multirow{2}{*}{$A S C_{2}$} & $a_{f}$ & $10 \mathrm{~mm}$ & $49 \mathrm{~mm}$ & $10 \mathrm{~mm}$ & $15.33 \mathrm{~mm}$ & \multirow{2}{*}{$105.2 \mathrm{~mm}$} \\
& $r_{t}$ & $3 \mathrm{~mm}$ & $25 \mathrm{~mm}$ & $6 \mathrm{~mm}$ & $3.3 \mathrm{~mm}$ & \\
\hline
\end{tabular}

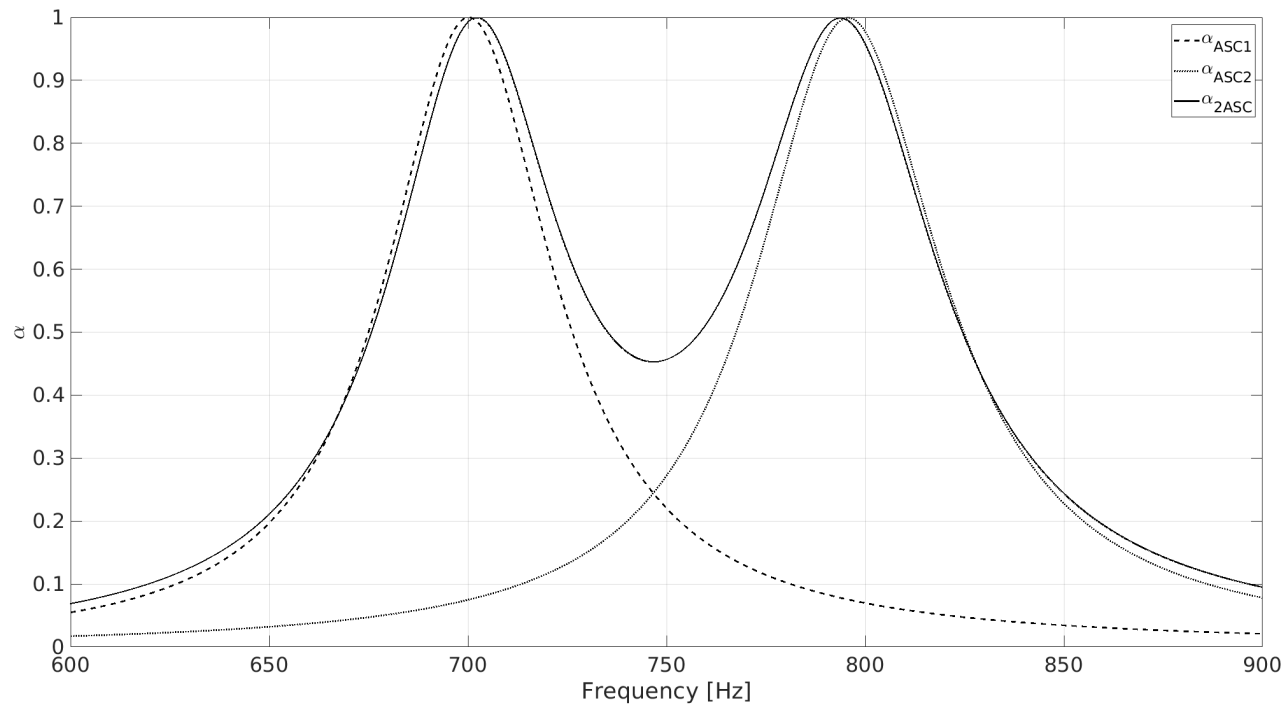

Figure 8: Theoretical results for 2ASC system.

The optimization delivers a maximum sound absorption coefficient at 700 $\mathrm{Hz}$ and $799.4 \mathrm{~Hz}$ for $\mathrm{ASC}_{1}$ and $\mathrm{ASC}_{2}$, respectively, as seen in Figure 8. When the two resonators are placed in parallel by using Equation (11), there is a small deviation of the peaks: from $700 \mathrm{~Hz}$ and $799.4 \mathrm{~Hz}$ to $702.2 \mathrm{~Hz}$ and 797.5 $\mathrm{Hz}$, respectively. The absorption coefficient at $700 \mathrm{~Hz}$ and $800 \mathrm{~Hz}$, which are the frequencies targeted by this system, are 0.9934 and 0.9928 , respectively, with almost zero reflection, thus showing that, even with the small deviation at the peaks due to the assembly, which affects the initial hypothesis of no interaction between the resonators, the 2ASC geometry performs almost 
full absorption at the frequencies for which it was designed. In addition, an impedance analysis, shown in Figure 9, reveals that the structure perfectly matches the impedance of the air at the optimized frequencies, since $\operatorname{Re}\left(Z / Z_{\text {air }}\right) \approx 1$ and $\operatorname{Im}\left(Z / Z_{\text {air }}\right) \approx 0$.
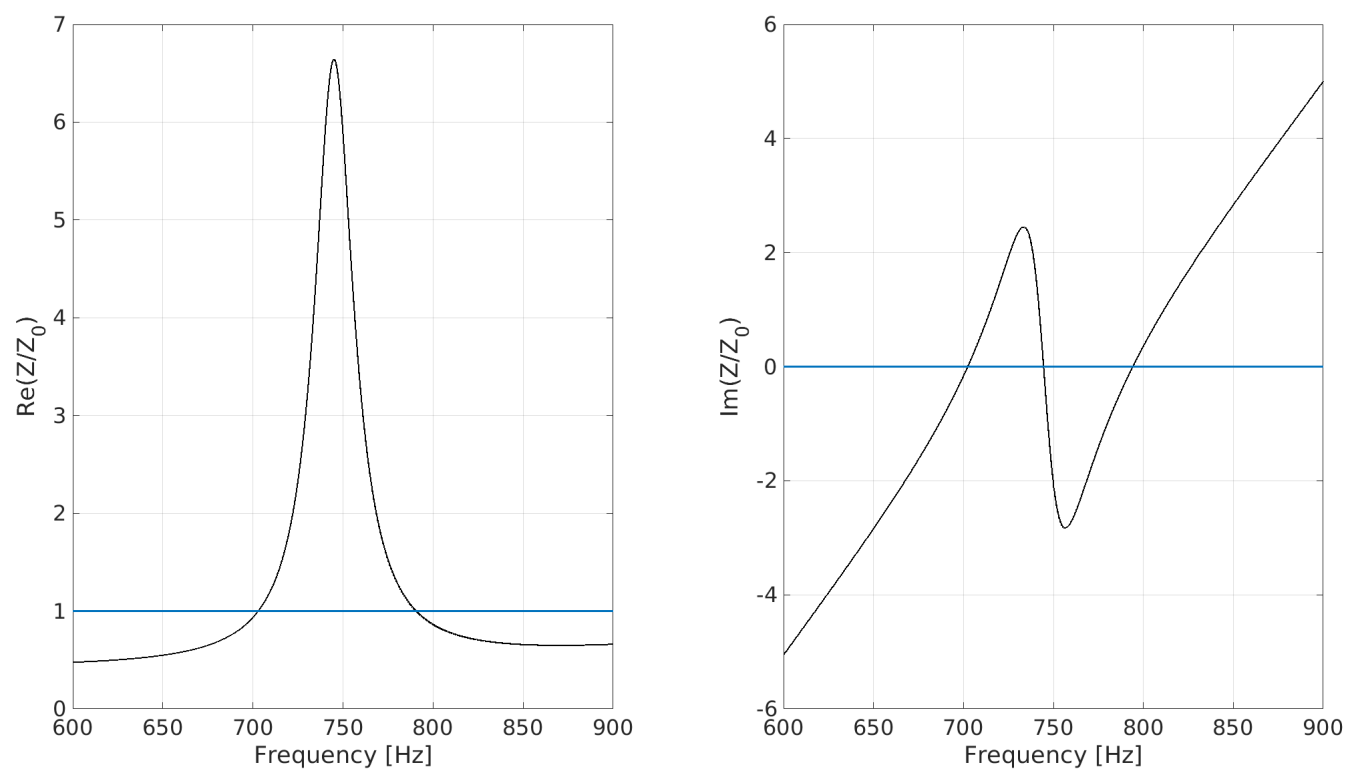

Figure 9: Impedance analysis of the 2ASC system.

\subsection{Numerical and experimental validation}

The analytical and FEA results, as well as the experimental ones, are shown in Figure 10. For the FEA analysis, two runs are performed: one considering the coiled channel or pore $(\mathrm{CP})$ and the other considering a straight pore (SP) with the same length, in order to assess any effects due to the coiling, which are reported in literature $[26,27]$ as a decrease in the effective propagation speed of the wave. From the simulations, it can be seen that this effect is small: in the lowest and highest peaks there is a shift from $702.5 \mathrm{~Hz}$ to $705 \mathrm{~Hz}$, and from $795 \mathrm{~Hz}$ to $792.5 \mathrm{~Hz}$, respectively. In terms of amount of absorption, at $700 \mathrm{~Hz}$ (lowest targeted frequency) it is 0.978 (SP) and $0.988(\mathrm{CP})$, therefore a change of only $\approx 1 \%$. As for the peak at $800 \mathrm{~Hz}$ (highest targeted frequency), the absorption values are 0.938 (SP) and 0.965 $(\mathrm{CP})$, a change of $\approx 2.7 \%$. 
The position of the peaks in both theoretical and numerical analysis match well, and the difference between these curves has a maximum of $\approx$ $4 \%$ at $\approx 747 \mathrm{~Hz}$. This might be due to some interaction effects, although small, as discussed previously, captured by the simulation.

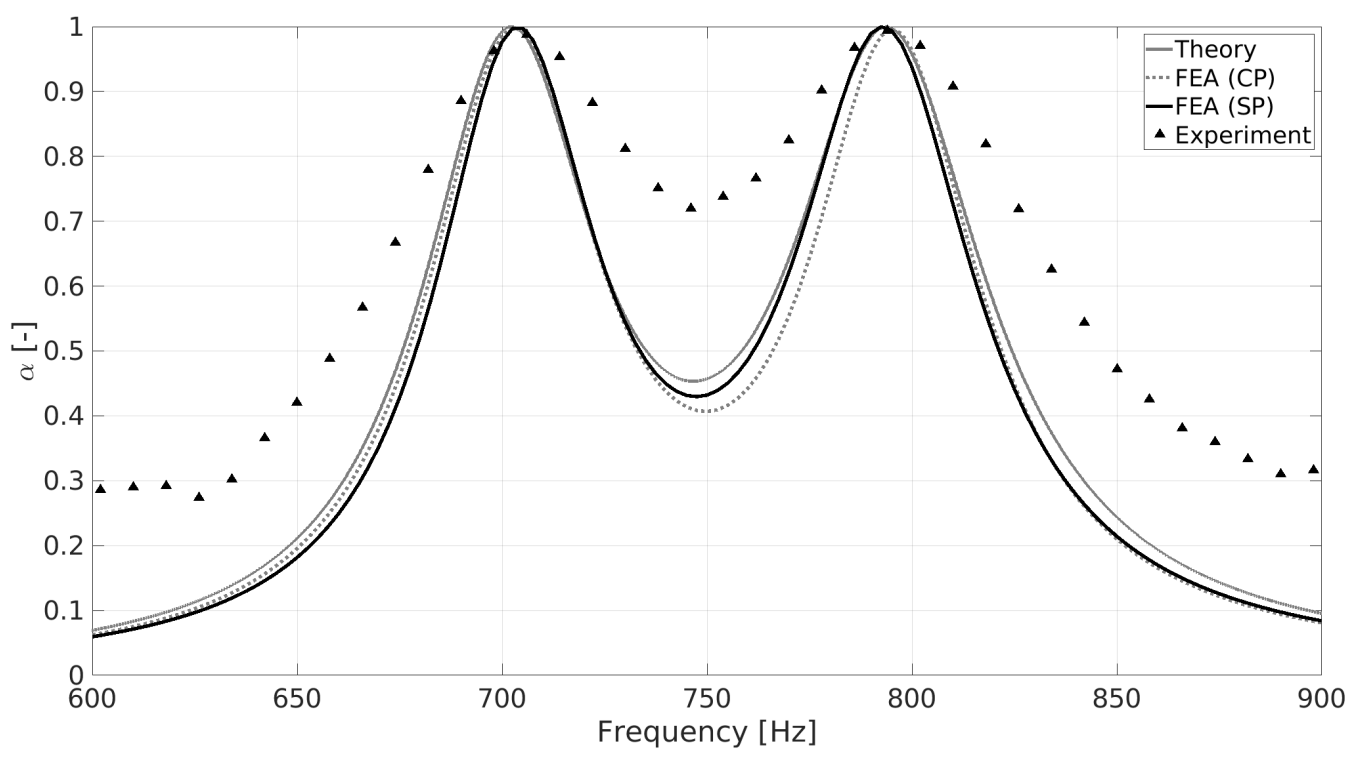

Figure 10: Experimental and numerical results for the 2ASC system.

The position of the experimental peaks have a fair match with the theoretical and numerical ones. Experimental peaks occur at $\approx 706 \mathrm{~Hz}$ and $\approx$ $794 \mathrm{~Hz}$, obtaining a total absorption of $98.73 \%$ and $99.33 \%$, respectively. The deviation of the experimental results from the predicted peaks (theory and FEA) might have been caused by geometrical inaccuracies of the 3D-printing machine. The total absorption at $700 \mathrm{~Hz}$ and $800 \mathrm{~Hz}$, the frequencies at which the system is tuned, are above $97 \%$ and $98 \%$, respectively. An overall $20 \%$ additional absorption is spotted between the two peaks as compared to the simulations and theory, which may indicate that effects of roughness are taking place, as well as the possibility of an existing air gap between the walls of the sample and the walls of the tube. Some papers argue that roughness of the sample might play a role $[28,29]$, however then both peaks would be expected to shift in the same direction.

The thickness of $18.4 \mathrm{~mm}$ of the sample represents $1 / 26.4$ of the wavelength at $706 \mathrm{~Hz}$ (experimental), thus making the unit cell a subwavelength 
absorptive structure. The length of the channel sets the lowest frequency that can be achieved with this kind of system. From Table 1, the length of each $\mathrm{ASC}$ is close to $\lambda / 4$ at their respective frequencies. If such length is to be increased, thus targeting a lower frequency, the diameter of the sample would need to be higher than $40 \mathrm{~mm}$, making experiments not possible since the tube available for testing has a diameter of $40 \mathrm{~mm}$. As such, with optimized values for $r_{t}$ and other parameters it is then feasible to obtain absorption for $f<<1000 \mathrm{~Hz}$, as seen in literature [10, 12], and have the system validated experimentally, provided a tube with feasible dimensions is available.

\section{Conclusion}

This paper presents a fully parameterized Archimedean spiral cavity that works as an acoustic resonator and that is 3D-printed in a fully modular way. It is also shown how to combine two of these ASCs in the same structure, with the aim of obtaining a compact geometry that provides unity sound absorption at more than one frequency for frequencies below $1000 \mathrm{~Hz}$. The system is validated through finite element analysis simulations as well as experimental results. A good agreement is found between experiments and theory, thus confirming the validity of the method.

\section{Acknowledgements}

The European Commission's ACOUTECT research and innovation programme under grant agreement No 721536 and the Research Fund KU Leuven are gratefully acknowledged for their support on this research. The research of E. Deckers is funded by a grant from the Research Foundation Flanders (FWO).

\section{References}

[1] D. A. Bies, C. Hansen, C. Howard, Engineering noise control, CRC press, 2017.

[2] F. Dunn, W. Hartmann, D. Campbell, N. H. Fletcher, Springer handbook of acoustics, Springer, 2015.

[3] T. Cox, P. d'Antonio, Acoustic absorbers and diffusers: theory, design and application, Crc Press, 2016. 
[4] D.-Y. Maa, Potential of microperforated panel absorber, the Journal of the Acoustical Society of America 104 (1998) 2861-2866.

[5] D.-Y. Maa, K. Liu, Sound absorption characteristics of microperforated absorber for random incidence, ACTA ACUSTICA-PEKING- 25 (2000) 289-296.

[6] K. Sakagami, Y. Nagayama, M. Morimoto, M. Yairi, Pilot study on wideband sound absorber obtained by combination of two different microperforated panel (mpp) absorbers, Acoustical science and technology 30 (2009) 154-156.

[7] K. Sakagami, S. Kobatake, K. Kano, M. Morimoto, M. Yairi, Sound absorption characteristics of a single microperforated panel absorber backed by a porous absorbent layer., Acoustics Australia 39 (2011).

[8] A. Komkin, M. Mironov, S. Yudin, On the attached length of orifices, Acoustical Physics 58 (2012) 628-632.

[9] A. Komkin, M. Mironov, A. Bykov, Sound absorption by a helmholtz resonator, Acoustical Physics 63 (2017) 385-392.

[10] X. Cai, Q. Guo, G. Hu, J. Yang, Ultrathin low-frequency sound absorbing panels based on coplanar spiral tubes or coplanar helmholtz resonators, Applied Physics Letters 105 (2014) 121901.

[11] Y. Li, B. M. Assouar, Acoustic metasurface-based perfect absorber with deep subwavelength thickness, Applied Physics Letters 108 (2016) 063502 .

[12] S. Huang, X. Fang, X. Wang, B. Assouar, Q. Cheng, Y. Li, Acoustic perfect absorbers via spiral metasurfaces with embedded apertures, Applied Physics Letters 113 (2018) 233501.

[13] C. Chen, Z. Du, G. Hu, J. Yang, A low-frequency sound absorbing material with subwavelength thickness, Applied Physics Letters 110 (2017) 221903.

[14] H. Ryoo, W. Jeon, Perfect sound absorption of ultra-thin metasurface based on hybrid resonance and space-coiling, Applied Physics Letters 113 (2018) 121903. 
[15] H. Ryoo, W. Jeon, Dual-frequency sound-absorbing metasurface based on visco-thermal effects with frequency dependence, Journal of Applied Physics 123 (2018) 115110.

[16] S.-H. Park, Acoustic properties of micro-perforated panel absorbers backed by helmholtz resonators for the improvement of low-frequency sound absorption, Journal of Sound and Vibration 332 (2013) 48954911.

[17] X.-L. Gai, T. Xing, X.-H. Li, B. Zhang, W.-J. Wang, Sound absorption of microperforated panel mounted with helmholtz resonators, Applied Acoustics 114 (2016) 260-265.

[18] S. Kim, Y.-H. Kim, J.-H. Jang, A theoretical model to predict the lowfrequency sound absorption of a helmholtz resonator array, The Journal of the Acoustical Society of America 119 (2006) 1933-1936.

[19] M. Yang, C. Meng, C. Fu, Y. Li, Z. Yang, P. Sheng, Subwavelength total acoustic absorption with degenerate resonators, Applied Physics Letters 107 (2015) 104104.

[20] X. Peng, J. Ji, Y. Jing, Composite honeycomb metasurface panel for broadband sound absorption, The Journal of the Acoustical Society of America 144 (2018) EL255-EL261.

[21] M. Yang, S. Chen, C. Fu, P. Sheng, Optimal sound-absorbing structures, Materials Horizons 4 (2017) 673-680.

[22] N. Jiménez, V. Romero-García, V. Pagneux, J.-P. Groby, Rainbowtrapping absorbers: Broadband, perfect and asymmetric sound absorption by subwavelength panels for transmission problems, Scientific Reports 7 (2017) 13595.

[23] E. W. Swokowski, Calculus with analytic geometry, Taylor \& Francis, 1979 .

[24] J. Allard, N. Atalla, Propagation of sound in porous media: modelling sound absorbing materials 2e, John Wiley \& Sons, 2009.

[25] M. R. Stinson, The propagation of plane sound waves in narrow and wide circular tubes, and generalization to uniform tubes of arbitrary 
cross-sectional shape, The Journal of the Acoustical Society of America 89 (1991) 550-558.

[26] X. Ni, Y. Wu, Z.-G. Chen, L.-Y. Zheng, Y.-L. Xu, P. Nayar, X.-P. Liu, M.-H. Lu, Y.-F. Chen, Acoustic rainbow trapping by coiling up space, Scientific reports 4 (2014) 7038.

[27] A. O. Krushynska, F. Bosia, N. M. Pugno, Labyrinthine acoustic metamaterials with space-coiling channels for low-frequency sound control, Acta Acustica United with Acustica 104 (2018) 200-210.

[28] S. Song, X. Yang, F. Xin, S. Ren, T. Lu, Modeling of roughness effects on acoustic properties of micro-slits, Journal of Physics D: Applied Physics 50 (2017) 235303.

[29] S. Song, X. Yang, F. Xin, T. J. Lu, Modeling of surface roughness effects on stokes flow in circular pipes, Physics of Fluids 30 (2018) 023604. 